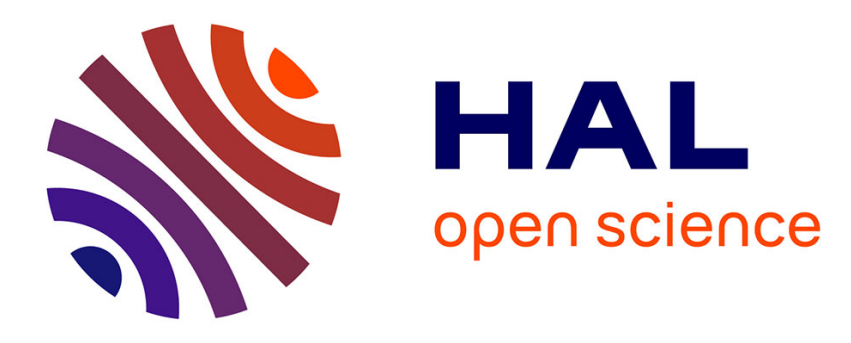

\title{
Epistasis, inbreeding depression and the evolution of self-fertilization
}

\author{
Diala Abu Awad, Denis Roze
}

\section{To cite this version:}

Diala Abu Awad, Denis Roze. Epistasis, inbreeding depression and the evolution of self-fertilization.

Peer Community in Evolutionary Biology, 2020, pp.100093. 10.1101/809814 . hal-02550660

\section{HAL Id: hal-02550660 \\ https://hal.sorbonne-universite.fr/hal-02550660}

Submitted on 22 Apr 2020

HAL is a multi-disciplinary open access archive for the deposit and dissemination of scientific research documents, whether they are published or not. The documents may come from teaching and research institutions in France or abroad, or from public or private research centers.
L'archive ouverte pluridisciplinaire HAL, est destinée au dépôt et à la diffusion de documents scientifiques de niveau recherche, publiés ou non, émanant des établissements d'enseignement et de recherche français ou étrangers, des laboratoires publics ou privés. 
Epistasis, inbreeding depression and the evolution of self-fertilization

Diala Abu Awad* and Denis Roze ${ }^{\dagger, \ddagger}$

* Department of Population Genetics, Technical University of Munich, Germany

† CNRS, UMI 3614 Evolutionary Biology and Ecology of Algae, 29688 Roscoff, France

‡ Sorbonne Université, Station Biologique de Roscoff, 29688 Roscoff, France 
Running title: Epistasis and selfing evolution

Keywords: epistasis, evolutionary quantitative genetics, inbreeding depression, multilocus population genetics, pollen discounting, self-fertilization

Address for correspondence:

Denis Roze

Station Biologique de Roscoff

Place Georges Teissier, CS90074

29688 Roscoff Cedex

France

Phone: (+33) 256452139

Fax: $(+33) 298292324$

email: roze@sb-roscoff.fr 


\section{ABSTRACT}

2 Inbreeding depression resulting from partially recessive deleterious alleles is

3 thought to be the main genetic factor preventing self-fertilizing mutants from spread-

4 ing in outcrossing hermaphroditic populations. However, deleterious alleles may also

5 generate an advantage to selfers in terms of more efficient purging, while the effects

6 of epistasis among those alleles on inbreeding depression and mating system evolution

7 remain little explored. In this paper, we use a general model of selection to disentangle

8 the effects of different forms of epistasis (additive-by-additive, additive-by-dominance

9 and dominance-by-dominance) on inbreeding depression and on the strength of se-

10 lection for selfing. Models with fixed epistasis across loci, and models of stabilizing

11 selection acting on quantitative traits (generating distributions of epistasis) are con-

12 sidered as special cases. Besides its effects on inbreeding depression, epistasis may

13 increase the purging advantage associated with selfing (when it is negative on aver-

14 age), while the variance in epistasis favors selfing through the generation of linkage

15 disequilibria that increase mean fitness. Approximations for the strengths of these

16 effects are derived, and compared with individual-based simulation results. 

and animals (e.g., Jarne and Auld, 2006; Igic and Busch, 2013). In Angiosperms, the transition from outcrossing to selfing occurred multiple times, leading to approximately $10-15 \%$ of species self-fertilizing at very high rates (Barrett et al., 2014). Two possible benefits of selfing have been proposed to explain such transitions: the possibility for a single individual to generate offspring in the absence of mating partner or pollinator 24 ("reproductive assurance", Darwin, 1876; Stebbins, 1957; Porcher and Lande, 2005a; Busch and Delph, 2012), and the "automatic advantage" stemming from the fact that, in a population containing both selfers and outcrossers, selfers tend to transmit more thus retaining the ability to sire outcrossed ovules (Fisher, 1941; Charlesworth, 1980; Stone et al., 2014). The main evolutionary force thought to oppose the spread of self- 
reducing the threshold value of inbreeding depression above which outcrossing can be

${ }_{41}$ maintained (e.g., Holsinger et al., 1984). It may also lead to evolutionarily stable 42 mixed mating systems (involving both selfing and outcrossing) under some models of 43 discounting such as the mass-action pollination model (Holsinger, 1991; Porcher and 44 Lande, 2005a).

Several models explored the evolution of mating systems while explicitly representing the genetic architecture of inbreeding depression (e.g., Charlesworth et al., 47 1990; Uyenoyama and Waller, 1991; Epinat and Lenormand, 2009; Porcher and Lande, 2005b; Gervais et al., 2014), and highlighted the importance of another genetic factor 49 (besides the automatic advantage and inbreeding depression) affecting the evolution of selfing. This third factor stems from the fact that selection against deleterious alleles is more efficient among selfed offspring (due to their increased homozygosity) than among outcrossed offspring, generating positive linkage disequilibria between alleles increas- 
have multiplicative effects (no epistasis). Charlesworth et al. (1991) considered a deterministic model including synergistic epistasis between deleterious alleles, showing that this form of epistasis tends to flatten the relation between inbreeding depression and the population's selfing rate, inbreeding depression sometimes increasing at high selfing rates. Concerning the spread of selfing modifier alleles, the results were qualitatively similar to the multiplicative model, except that, for parameter values where full outcrossing is not stable, the evolutionarily stable selfing rate tended to be slightly below 1 under synergistic epistasis (whereas it would have been at exactly 1 in the absence of epistasis). Other models explored the effect of partial selfing on inbreeding depression generated by polygenic quantitative traits under stabilizing selection (Lande and Porcher, 2015; Abu Awad and Roze, 2018). This type of model typically generates distributions of epistatic interactions across loci, including possible compensatory effects between mutations. When effective recombination is sufficiently weak, linkage disequilibria generated by epistasis may greatly reduce inbreeding depression, and even generate outbreeding depression between selfing lineages carrying different combinations of compensatory mutations. However, the evolution of the selfing rate was not considered by these models.

In this paper, we use a general model of epistasis between pairs of selected loci to explore the effects of epistasis on inbreeding depression and on the evolution of selfing. We derive analytical approximations showing that epistatic interactions affect the spread of selfing modifiers through various mechanisms: by affecting inbreeding depression, the purging advantage of selfers and also through linkage disequilibria between selected loci. Although the expressions obtained can become complicated for intermediate selfing rates, we will see that the condition determining whether selfing can spread 
in a fully outcrossing population often remains relatively simple. Notably, our model allows us to disentangle the effects of additive-by-additive, additive-by-dominance and dominance-by-dominance epistatic interactions on inbreeding depression and selection for selfing — while the models used by Charlesworth et al. (1991), Lande and Porcher (2015) and Abu Awad and Roze (2018) impose certain relations between these quantities. The cases of fixed, synergistic epistasis and of stabilizing selection acting on quantitative traits (Fisher's geometric model) will be considered as special cases, for which we will also present individual-based simulation results. Overall, our results show that, for a given level of inbreeding depression and average strength of selection against deleterious alleles, epistatic interactions tend to facilitate the spread of selfing, due to the fact that selfing can maintain beneficial combinations of alleles.

Life cycle. Our analytical model represents an infinite, hermaphroditic population with discrete generations. A proportion $\sigma$ of ovules produced by a given individual are self-fertilized, while its remaining ovules are fertilized by pollen sampled from the population pollen pool (Table 1 provides a list of the symbols used throughout the paper). A parameter $\kappa$ represents the rate of pollen discounting: an individual with selfing rate $\sigma$ contributes to the pollen pool in proportion $1-\kappa \sigma$ (e.g., Charlesworth, 1980). Therefore, $\kappa$ equals 0 in the absence of pollen discounting, while $\kappa$ equals 1 under full discounting (in which case complete selfers do not contribute to the pollen pool). We assume that the selfing rate $\sigma$ is genetically variable, and coded by $\ell_{\sigma}$ loci 
with additive effects:

$$
\sigma=\sum_{i=1}^{\ell_{\sigma}}\left(\sigma_{i}^{\mathrm{M}}+\sigma_{i}^{\mathrm{P}}\right)
$$

The fitness $W$ of an organism is defined as its overall fecundity (that may depend on its survival), so that the expected number of seeds produced by an individual is proportional to $W$, while its contribution to the population pollen pool is proportional to $W(1-\kappa \sigma)$. We assume that $W$ is affected by a possibly large number $\ell$ of biallelic loci. Alleles at each of these loci are denoted 0 and $1 ;$ the quantity $X_{j}^{\mathrm{M}}\left(\operatorname{resp} . X_{j}^{\mathrm{P}}\right)$ equals 0 if the individual carries allele 0 on its maternally (resp. paternally) inherited copy of locus $j$, and equals 1 otherwise. The frequencies of allele 1 at locus $j$ on the maternally and paternally inherited genes (averages of $X_{j}^{\mathrm{M}}$ and $X_{j}^{\mathrm{P}}$ over the whole population) are denoted $p_{j}^{\mathrm{M}}$ and $p_{j}^{\mathrm{P}}$. Finally, $p_{j}=\left(p_{j}^{\mathrm{M}}+p_{j}^{\mathrm{P}}\right) / 2$ is the frequency of allele 1 at locus $j$ in the whole population.

Genetic associations. Throughout the paper, index $i$ will denote a locus affecting the selfing rate of individuals, while indices $j$ and $k$ will denote loci affecting fitness. Following Barton and Turelli (1991) and Kirkpatrick et al. (2002), we define the centered variables:

$$
\zeta_{i}^{\mathrm{M}}=\sigma_{i}^{\mathrm{M}}-\overline{\sigma_{i}^{\mathrm{M}}}, \quad \zeta_{i}^{\mathrm{P}}=\sigma_{i}^{\mathrm{P}}-\overline{\sigma_{i}^{\mathrm{P}}}
$$




$$
\zeta_{j}^{\mathrm{M}}=X_{j}^{\mathrm{M}}-p_{j}^{\mathrm{M}}, \quad \zeta_{j}^{\mathrm{P}}=X_{j}^{\mathrm{P}}-p_{j}^{\mathrm{P}},
$$

130 where $\overline{\sigma_{i}^{\mathrm{M}}}$ and $\overline{\sigma_{i}^{\mathrm{P}}}$ are the averages of $\sigma_{i}^{\mathrm{M}}$ and $\sigma_{i}^{\mathrm{P}}$ over the whole population. The comes:

$$
V_{\sigma} \approx 2 \sum_{i}\left(\tilde{D}_{i i}+D_{i, i}\right)
$$

${ }_{142}$ General expression for fitness, and special cases. The fitness of an individual 143 divided by the population mean fitness $\bar{W}$ can be expressed in terms of "selection coefficients" $a_{\mathbb{U}, \mathbb{V}}$ representing the effect of selection acting on the sets $\mathbb{U}$ and $\mathbb{V}$ of loci 
(Barton and Turelli, 1991; Kirkpatrick et al., 2002):

$$
\frac{W}{\bar{W}}=1+\sum_{\mathbb{U}, \mathbb{V}} a_{\mathbb{U}, \mathbb{V}}\left(\zeta_{\mathbb{U}, \mathbb{V}}-D_{\mathbb{U}, \mathbb{V}}\right)
$$

146

163

We will consider different examples of fitness functions (for which approximate expressions for $a_{\mathbb{U}, \mathbb{V}}$ coefficients are given in Supplementary File S1). The first corresponds to the case where allele 1 at each fitness locus $j$ is deleterious, with selection and dominance coefficients $s$ and $h$. Epistatic interactions occur between pairs of loci, and are decomposed into additive-by-additive $\left(e_{\text {axa }}\right)$, additive-by-dominance $\left(e_{\text {axd }}\right)$ and dominance-by-dominance $\left(e_{\mathrm{dxd}}\right)$ epistasis. We assume multiplicative effects of epistatic components on fitness $W$ (i.e., additive effects on $\log W$ ), so that:

$$
W=(1-h s)^{n_{\mathrm{he}}}(1-s)^{n_{\mathrm{ho}}}\left(1+e_{\mathrm{axa}}\right)^{n_{2}}\left(1+e_{\mathrm{axd}}\right)^{n_{3}}\left(1+e_{\mathrm{dxd}}\right)^{n_{4}}
$$

where $n_{\text {he }}$ and $n_{\text {ho }}$ are the numbers of loci at which a deleterious allele is present in the heterozygous $\left(n_{\text {he }}\right)$ or homozygous $\left(n_{\text {ho }}\right)$ state, while $n_{2}, n_{3}$ and $n_{4}$ are the numbers of interactions between 2, 3 and 4 deleterious alleles at two different loci, given by:

$$
n_{2}=\frac{1}{2} n_{\mathrm{he}}\left(n_{\mathrm{he}}-1\right)+2 n_{\mathrm{he}} n_{\mathrm{ho}}+2 n_{\mathrm{ho}}\left(n_{\mathrm{ho}}-1\right)
$$

$$
n_{3}=n_{\mathrm{he}} n_{\mathrm{ho}}+2 n_{\mathrm{ho}}\left(n_{\mathrm{ho}}-1\right)
$$




$$
n_{4}=\frac{1}{2} n_{\mathrm{ho}}\left(n_{\mathrm{ho}}-1\right) .
$$

165

$$
W=\exp \left[-\frac{\sum_{\alpha=1}^{n} g_{\alpha}{ }^{2}}{2 V_{\mathrm{s}}}\right]
$$


where $V_{\mathrm{s}}$ represents the strength of selection. According to equation 14, the optimal value of each trait is zero. This model generates distributions of fitness effects of mutations and of pairwise epistatic effects on fitness (the average value of epistasis being zero), while deleterious alleles have a dominance coefficient close to $1 / 4$ in an optimal genotype (Martin and Lenormand, 2006b; Martin et al., 2007; Manna et al., 2011).

The last fitness function we examined is a generalization of the fitness function given by equation 14, in order to introduce a coefficient $Q$ affecting the shape of the fitness peak:

$$
W=\exp \left[-\left(\frac{d}{\sqrt{2 V_{\mathrm{s}}}}\right)^{Q}\right],
$$

where $d=\sqrt{\sum_{\alpha=1}^{n} g_{\alpha}^{2}}$ is the Euclidean distance from the optimum in phenotypic space (e.g., Martin and Lenormand, 2006a; Tenaillon et al., 2007; Roze and Blanckaert, 2014; Abu Awad and Roze, 2018). The fitness function is thus Gaussian when $Q=2$, while $Q>2$ leads to a flatter fitness peak around the optimum. As shown by Gros et al. (2009), the value of $Q$ affects the average value of epistasis (on fitness) between mutations, which becomes negative when $Q>2$.

Quasi-linkage equilibrium (QLE) approximation. Using the general expression for fitness given by equation 8 , the change in the mean selfing rate per generation can be expressed in terms of genetic associations between loci affecting the selfing rate and loci affecting fitness. Expressions for these associations can then be computed using general methods to derive recursions on allele frequencies and genetic associations (Barton and Turelli, 1991; Kirkpatrick et al., 2002). For this, we decompose the life cycle into two steps: selection corresponds to the differential contribution of individuals 
due to differences in overall fecundity and/or survival rates $(W)$, while reproduction corresponds to gamete production and fertilization (involving either selfing or outcrossing). Associations measured after selection (that is, weighting each parent by its relative fitness) will be denoted $D_{\mathbb{U}, \mathbb{V}}^{\prime}$, while associations after reproduction (among offspring) will be denoted $D_{\mathbb{U}, \mathbb{V}}^{\prime \prime}$. Assuming that "effective recombination rates" (that is, recombination rates multiplied by outcrossing rates) are sufficiently large relative to the strength of selection, genetic associations equilibrate rapidly relative to the change in allele frequencies due to selection. In that case, associations can be expressed in terms of allele frequencies by computing their values at equilibrium, for given allele frequencies (e.g., Barton and Turelli, 1991; Nagylaki, 1993). Note that when allele frequencies at fitness loci have reached an equilibrium (for example, at mutation-selection balance), one does not need to assume that the selection coefficients $a_{\mathbb{U}, \mathbb{V}}$ are small relative to effective recombination rates for the QLE approximation to hold, but only that changes in allele frequencies due to the variation in the selfing rate between individuals are small. We will thus assume that the variance in the selfing rate in the population $V_{\sigma}$ stays small (and therefore, the genetic variance contributed by each locus affecting the selfing rate is also small), and compute expressions to the first order in $V_{\sigma}$. This is equivalent to the assumption that alleles at modifier loci have small effects, as is commonly assumed in modifier models.

Individual-based simulations. In order to verify our analytical results, individualbased simulations were run using two $\mathrm{C}++$ programs, one with uniformly deleterious alleles with fixed epistatic effects (equation 9) and the other with stabilizing selection on $n$ quantitative traits (equation 14). Both are described in Supplementary File S5 
(and are available from Dryad). Both programs represent a population of $N$ diploid individuals with discrete generations, the genome of each individual consisting of two copies of a linear chromosome with map length $R$ Morgans. In the first program (fixed epistasis), deleterious alleles occur at rate $U$ par haploid genome per generation at an infinite number of possible sites along the chromosome. A locus with an infinite number of possible alleles, located at the mid-point of the chromosome controls the selfing rate of the individual (given by averaging the selfing rate coded by the two alleles at this locus). In the program representing stabilizing selection, each chromosome carries $\ell$ equidistant biallelic loci affecting the $n$ traits under selection (as in Abu Awad and Roze, 2018). The selfing rate is controlled by 10 additive loci evenly spaced over the chromosome, each with an infinite number of possible alleles (the selfing rate being set to zero if the sum of allelic values at these loci is negative, and one if the sum is larger than one). In both programs, mutations affecting the selfing rate occur at rate $U_{\text {self }}=10^{-3}$ per generation, the value of each mutant allele at a selfing modifier locus being drawn from a Gaussian distribution with standard deviation $\sigma_{\text {self }}$ centered on the allele value before mutation. The selfing rate is set to zero during an initial burn-in period (set to 20,000 generations) after which mutations are introduced at selfing modifier loci.

Effects of epistasis on inbreeding depression. We first explore the effects of epistasis on inbreeding depression, assuming that the selfing rate is fixed. Throughout 
the paper, inbreeding depression $\delta$ is classically defined as:

$$
\delta=1-\frac{\bar{W}^{\text {self }}}{\bar{W}^{\text {out }}}
$$

${ }_{251}$ where $\bar{W}^{\text {self }}$ and $\bar{W}^{\text {out }}$ are the mean fitnesses of offspring produced by selfing and by

$$
\delta \approx-\frac{1}{2} \sum_{j} a_{j, j} p_{j} q_{j}-\frac{1}{2} \sum_{j<k} a_{j k, j k}\left[1-2 \rho_{j k}\left(1-\rho_{j k}\right)\right] p_{j} q_{j} p_{k} q_{k}-\sum_{j<k} c_{j k} \tilde{D}_{j k}
$$

5 where the sums are over all loci affecting fitness, and with:

$$
c_{j k}=a_{j, k}+\left[a_{j k, j}\left(1-2 p_{j}\right)+a_{j k, k}\left(1-2 p_{k}\right)\right]\left(1-\rho_{j k}\right),
$$

$$
F=\frac{\sigma}{2-\sigma}
$$

${ }_{263}$ at equilibrium, while $G_{j k}$ is the identity disequilibrium between loci $j$ and $k$ (Weir and ${ }_{264}$ Cockerham, 1973), given by:

$$
G_{j k}=\phi_{j k}-F^{2}, \quad \text { with } \quad \phi_{j k}=\frac{\sigma}{2-\sigma} \frac{2-\sigma-2(2-3 \sigma) \rho_{j k}\left(1-\rho_{j k}\right)}{2-\sigma\left[1-2 \rho_{j k}\left(1-\rho_{j k}\right)\right]}
$$




$$
\begin{aligned}
\Delta_{\text {sel }} p_{j} \approx & a_{j}(1+F) p_{j}+a_{j, j} F p_{j}+\sum_{k \neq j} a_{j k, k}\left[F(1+F)+G_{j k}\right] p_{j} p_{k} \\
& +\sum_{k \neq j} a_{j k, j k}\left[F^{2}+G_{j k}\right] p_{j} p_{k} .
\end{aligned}
$$

which will be denoted $G$ hereafter.

In the case of unconditionally deleterious alleles with fixed epistasis (equation 9), equation 19 and the expressions for $a_{\mathbb{U}, \mathbb{V}}$ coefficients given in Supplementary File

$$
\delta \approx 1-\exp \left[-\frac{1}{2}\left[s(1-2 h)-2 e_{\mathrm{axd}} n_{\mathrm{d}}\right](1+F) n_{\mathrm{d}}+\frac{e_{\mathrm{dxd}}}{8}\left[(1+F)^{2}+G\right] n_{\mathrm{d}}^{2}\right]
$$

$$
\Delta_{\text {sel }} n_{\mathrm{d}}+U=0
$$

279 Summing over loci and using the expressions for $a_{\mathbb{U}, \mathbb{V}}$ coefficients given in Supplemen- 
tary File S1, one obtains:

$$
\begin{aligned}
\Delta_{\text {sel }} n_{\mathrm{d}} \approx & -s[h+(1-h) F] n_{\mathrm{d}}+2 e_{\text {axa }}(1+F) n_{\mathrm{d}}^{2} \\
& +e_{\text {axd }}[F(3+F)+G] n_{\mathrm{d}}^{2}+e_{\mathrm{dxd}}\left(F^{2}+G\right) n_{\mathrm{d}}^{2}
\end{aligned}
$$

that can be used with equation 24 to obtain the equilibrium value of $n_{\mathrm{d}}$. Equation 26 shows that, for non-random mating, negative values of $e_{\mathrm{axa}}, e_{\mathrm{axd}}$ or $e_{\mathrm{dxd}}$ reduce the mean number of deleterious alleles at equilibrium, thereby reducing inbreeding depression (the effects of $e_{\mathrm{axd}}$ and $e_{\mathrm{dxd}}$ on the equilibrium value of $n_{\mathrm{d}}$ vanish when mating is random, as $F=G=0$ in this case). As shown by equation 23 , negative values of $e_{\mathrm{axd}}$ and $e_{\mathrm{dxd}}$ also directly increase inbreeding depression (even under random mating), by decreasing the fitness of homozygous offspring. Figures $1 \mathrm{~A}-\mathrm{C}$ compare the predictions obtained from equations 23 and 26 with simulation results, testing the effect of each epistatic component separately. Negative $e_{\text {axa }}$ reduces inbreeding depression by lowering the frequency of deleterious alleles in the population (equation 26, Figure 1A); furthermore, it reduces the purging effect of selfing, so that inbreeding depression may remain constant or even slightly increase as the selfing rate increases. When the selfing rate is low, $e_{\mathrm{axd}}$ and $e_{\mathrm{dxd}}$ have little effect on the mean number of deleterious alleles $n_{\mathrm{d}}$, and the main effect of negative $e_{\mathrm{axd}}$ and $e_{\mathrm{dxd}}$ is to increase inbreeding depression by decreasing the fitness of homozygous offspring (equation 23, Figures $1 \mathrm{~B}-\mathrm{C})$. As selfing increases, this effect becomes compensated by the enhanced purging caused by negative $e_{\mathrm{axd}}$ and $e_{\mathrm{dxd}}$ (equation 26). Figure 1D shows the results obtained using Charlesworth et al.'s (1991) fitness function, yielding $e_{\text {axa }}=-\beta h^{2}$, $e_{\mathrm{axd}}=-\beta h(1-2 h)$ and $e_{\mathrm{dxd}}=-\beta(1-2 h)^{2}$. Remarkably, the increased purging caused by negative epistasis almost exactly compensates the decreased fitness of homozygous offspring, so that inbreeding depression is only weakly affected by epistasis 
in this particular model, for the parameter values used in Figure 1.

An expression for inbreeding depression under Gaussian stabilizing selection (equation 14) is given in Abu Awad and Roze (2018). As shown in Supplementary File S2, this expression can be recovered from our general expression for $\delta$ in terms of $a_{\mathbb{U}, \mathbb{V}}$ coefficients. Because the average epistasis is zero under Gaussian selection (e.g., Martin et al., 2007), inbreeding depression is only affected by the variance in epistasis, whose main effect is to generate linkage disequilibria that increase the frequency of deleterious alleles (see also Phillips et al., 2000) and thus increase $\delta$. As shown by Abu Awad and Roze (2018), a different regime is entered above a threshold selfing rate when the mutation rate $U$ is sufficiently large, in which epistatic interactions lower inbreeding depression (see also Lande and Porcher, 2015). Selection coefficients $a_{\mathbb{U}, \mathbb{V}}$ under the more general fitness function given by equation 15 are derived in Supplementary File S1, showing that a "flatter-than-Gaussian" fitness peak $(Q>2)$ generates negative dominance-by-dominance epistasis $\left(a_{j k, j k}<0\right)$, increasing inbreeding depression (by contrast, the first term of equation 17 representing the effect of dominance is not affected by $Q$ ). In the absence of selfing, and neglecting the effects of genetic associations among loci, one obtains (see Supplementary File S2 for derivation):

$$
\delta \approx 1-\exp \left[-U\left(1+\frac{Q-2}{8}\right)\right]
$$

where the term in $(Q-2) / 8$ is generated by the term in $a_{j k, j k}$ in equation 17 . Although this expression differs from equation 29 in Abu Awad and Roze (2018) — that was obtained using a different method — both results are quantitatively very similar as long as $Q$ is not too large (roughly, $Q<6$ ). Generalizations of equation 27 to arbitrary $\sigma$, and including the effects of pairwise associations between loci (for $\sigma=0$ ) are given 
in Supplementary File S2 (equations B40 and B54).

Evolution of selfing in the absence of epistasis. In Supplementary File S3, we derive an expression for the change in the mean selfing rate $\bar{\sigma}$ per generation, neglecting the effects of epistatic interactions and associations between loci affecting fitness. This expression can be decomposed into three terms:

$$
\Delta \bar{\sigma}=\Delta_{\text {auto }} \bar{\sigma}+\Delta_{\text {depr }} \bar{\sigma}+\Delta_{\text {purge }} \bar{\sigma}
$$

with:

$$
\Delta_{\text {auto }} \bar{\sigma} \approx \frac{1-\kappa}{1-\kappa \bar{\sigma}} \frac{V_{\sigma}^{\prime}}{2}
$$

$$
\Delta_{\mathrm{depr}} \bar{\sigma}=2 \sum_{i, j} a_{j, j} \tilde{D}_{i j, j}
$$

$$
\Delta_{\text {purge }} \bar{\sigma}=2 \sum_{i, j} a_{j}\left(\tilde{D}_{i j}+\tilde{D}_{i, j}\right)
$$

where the sums are over all loci $i$ affecting the selfing rate and all loci $j$ affecting fitness.

The term $\Delta_{\text {auto }} \bar{\sigma}$ represents selection for increased selfing rates due to the automatic transmission advantage associated with selfing (Fisher, 1941). It is proportional to the variance in selfing rate after selection $V_{\sigma}^{\prime}$, and vanishes when pollen discounting is complete $(\kappa=1)$. The second term corresponds to the effect of inbreeding depression. It depends on coefficients $a_{j, j}$ representing the effect of dominance at loci affecting fitness; in particular, $a_{j, j}<0$ when the average fitness of the two homozygotes at locus $j$ is lower than the fitness of heterozygotes (which is the case when the deleterious allele at locus $j$ is recessive or partially recessive). It also depends on associations $\tilde{D}_{i j, j}$ that are shown to be positive at QLE, reflecting the fact that alleles increasing the selfing rate tend to be present on more homozygous backgrounds. Finally, the last term depends on coefficients $a_{j}$ representing directional selection for allele 1 at locus 
359

$$
\tilde{D}_{i j} \approx \frac{1}{2} \frac{a_{j}+a_{j, j}\left(1-2 p_{j}\right)}{\rho_{i j}-a_{j}\left(1-2 p_{j}\right)\left(1-\rho_{i j}\right)} \tilde{D}_{i i} p_{j} q_{j}, \quad \tilde{D}_{i, j} \approx 0 .
$$

360 361

$$
\Delta_{\text {purge }} \bar{\sigma} \approx \sum_{j}\left[\mathcal{E}\left[\frac{1}{\rho_{i j}-a_{j}\left(1-2 p_{j}\right)\left(1-\rho_{i j}\right)}\right] a_{j}\left[a_{j}+a_{j, j}\left(1-2 p_{j}\right)\right] p_{j} q_{j}\right] \frac{V_{\sigma}}{2}
$$

Using the fact that $V_{\sigma}=2 \sum_{i} \tilde{D}_{i i}$ under random mating (equation 7), equations $29-$ 33 yield, for $\bar{\sigma} \approx 0$ :

$$
\Delta_{\mathrm{auto}} \bar{\sigma} \approx \frac{1-\kappa}{2} V_{\sigma}, \quad \Delta_{\mathrm{depr}} \bar{\sigma} \approx-\delta V_{\sigma}
$$

where $\delta=-\left(\sum_{j} a_{j, j} p_{j} q_{j}\right) / 2$ is inbreeding depression, neglecting the effect of interactions between selected loci (see equation 17), while 
where the sum is over all loci $j$ affecting fitness, and where $\mathcal{E}$ is the average over all loci $i$ affecting the selfing rate. Because $\Delta_{\text {purge }} \bar{\sigma}$ is of second order in the selection coefficients $\left(a_{j}, a_{j, j}\right)$, it will generally be negligible relative to $\Delta_{\text {depr }} \bar{\sigma}$ (which is of first order in $\left.a_{j, j}\right)$, in which case selfing can increase if $\delta<(1-\kappa) / 2$ (Charlesworth, 1980). When $\bar{\sigma}>0, \Delta_{\text {depr }} \bar{\sigma}$ is not simply given by $\delta V_{\sigma}$ (in particular, it also depends on the rate of pollen discounting and on identity disequilibria between loci affecting the selfing rate and loci affecting fitness, as shown by equation C31 in Supplementary File S3), but it is possible to show that $\Delta_{\text {depr }} \bar{\sigma}$ tends to decrease in magnitude as $\bar{\sigma}$ increases (while $\Delta_{\text {auto }} \bar{\sigma}$ becomes stronger as $\bar{\sigma}$ increases), leading to the prediction that $\bar{\sigma}=0$ and $\bar{\sigma}=1$ should be the only evolutionarily stable selfing rates (Lande and Schemske, 1985).

As shown by equation 35 , the relative importance of $\Delta_{\text {purge }} \bar{\sigma}$ should increase when the strength of directional selection $\left(a_{j}\right)$ increases, when deviations from additivity $\left(a_{j, j}\right)$ are weaker and when linkage among loci is tighter. In the case where allele 1 at each fitness locus is deleterious with selection and dominance coefficients $s$ and $h$ (and assuming that $p_{j} \ll 1$ ) we have $a_{j} \approx-s h$ and $a_{j, j} \approx-s(1-2 h$ ), while $p_{j} q_{j} \approx u /(s h)$ at mutation-selection balance (where $u$ is the per locus mutation rate towards allele 1). In that case, equation 35 simplifies to:

$$
\Delta_{\text {purge }} \bar{\sigma} \approx \mathcal{E}\left[\frac{1}{\rho_{i j}+s h\left(1-\rho_{i j}\right)}\right] s(1-h) U \frac{V_{\sigma}}{2}
$$

where $U$ is the deleterious mutation rate per haploid genome and $\mathcal{E}$ is now the average over all pairs of loci $i$ and $j$. Figure 2A compares the prediction obtained from equations 34 and 36 with simulation results, in the absence of pollen discounting $(\kappa=0)$, and when alleles affecting the selfing rate have weak effects $\left(\sigma_{\text {self }}=0.01\right)$. Simulations 
confirm that selfing may evolve when inbreeding depression is higher than 0.5 (due to the effect of $\left.\Delta_{\text {purge }} \bar{\sigma}\right)$, provided that the fitness effect of deleterious alleles is sufficiently strong. The prediction for the case of unlinked loci (obtained by setting $\rho_{i j}=0.5$ in equation 36) actually gives a closer match to the simulation results than the result obtained by integrating equation 36 over the genetic map. This may stem from the fact that equation 36 overestimates the effect of tightly linked loci. The effect of the size of mutational steps at the modifier locus does not affect the maximum value of inbreeding depression for which selfing can spread, as long as mutations tend to have small effects on the selfing rate (compare Figure 2A and 2B). However, the relative effect of purging (observed for high values of $s$ ) becomes more important when selfing evolves by mutations of larger size $\left(\sigma_{\text {self }}=0.3\right.$ in Figure $2 \mathrm{C}$, while mutations directly lead to fully selfing individuals in Figure 2D), in agreement with the results obtained by Charlesworth et al. (1990) — note that our approximations break down when selfing evolves by large-effect mutations.

In the case of multivariate Gaussian stabilizing selection acting on $n$ traits coded by biallelic loci with additive effects (equation 14) we have (to the first order in the strength of selection $\left.1 / V_{\mathrm{s}}\right): a_{j}=-\varsigma_{j}\left(1-2 p_{j}\right)$ and $a_{j, j}=-2 \varsigma_{j}$, where $\varsigma_{j}=$ $\sum_{\alpha=1}^{n} r_{\alpha j}^{2} /\left(2 V_{\mathrm{s}}\right)$ is the fitness effect of a heterozygous mutation at locus $j$ in an optimal genotype. Assuming that polymorphism stays weak at loci coding for the traits under stabilizing selection, so that $\left(1-2 p_{j}\right)^{2} \approx 1$, and using the fact that $p_{j} q_{j} \approx u / \varsigma_{j}$ under random mating (when neglecting interactions between loci), one obtains from equation 35 :

$$
\Delta_{\text {purge }} \bar{\sigma} \approx \mathcal{E}\left[\frac{3 \varsigma_{j}}{\rho_{i j}+\varsigma_{j}\left(1-\rho_{i j}\right)}\right] U \frac{V_{\sigma}}{2}
$$

408 which is equivalent to equation 36 when introducing differences in $s$ among loci, with 
$h=1 / 4$ (note that the homozygous effect of mutation at locus $j$ in an optimal genotype is $\approx 4 \varsigma_{j}$ ). When neglecting the term in $\varsigma_{j}$ in the denominator of equation 37 , this simplifies to:

$$
\Delta_{\text {purge }} \bar{\sigma} \approx \frac{3}{2} \frac{\bar{\varsigma} U V_{\sigma}}{\rho_{\mathrm{h}, \sigma z}}
$$

where $\bar{\varsigma}$ is the mean heterozygous effect of mutations on fitness in an optimal genotype, and where $\rho_{\mathrm{h}, \sigma z}$ is the harmonic mean recombination rate over all pairs of loci $i$ and $j$, where $i$ affects the selfing rate and $j$ affects the traits under stabilizing selection. Using the fitness function given by equation 15 (where $Q$ describes the shape of the fitness peak), equation 38 generalizes to:

$$
\Delta_{\text {purge }} \bar{\sigma} \approx \frac{3 U^{2}}{\rho_{\mathrm{h}, \sigma z}}\left(\frac{4 U}{Q \bar{\varsigma}}\right)^{-\frac{2}{Q}} V_{\sigma}
$$

(see Supplementary File S1), which increases as $Q$ increases in most cases. Therefore, for a given value of inbreeding depression, a flatter fitness peak tends to increase the relative importance of purging on the spread of selfing mutants in an outcrossing population.

Effects of epistasis on the evolution of selfing. Expressions for the change in mean selfing rate $\bar{\sigma}$, including the effects of epistasis between pairs of selected loci are derived in Supplementary File S4. Because the expressions quickly become cumbersome under partial selfing, we restrict our analysis to the initial spread of selfing ${ }_{225}$ in an outcrossing population $(\bar{\sigma} \approx 0)$. The change in mean selfing rate per generation 26 now writes:

$$
\Delta \bar{\sigma}=\Delta_{\text {auto }} \bar{\sigma}+\Delta_{\text {depr }} \bar{\sigma}+\Delta_{\mathrm{LD}} \bar{\sigma}+\Delta_{\text {purge }} \bar{\sigma}
$$

${ }_{427}$ As above, $\Delta_{\text {auto }} \bar{\sigma}$ represents the direct transmission advantage of selfing and is still 
epistasis (besides its effects on inbreeding depression $\delta^{\prime}$ ), and is given by:

$$
\Delta_{\mathrm{LD}} \bar{\sigma}=2 \sum_{i, j<k} a_{j k} \tilde{D}_{i j k} .
$$

${ }_{446}$ The association $\tilde{D}_{i j k}$ represents the fact that the linkage disequilibrium $D_{j k}$ between ${ }_{447}$ loci $j$ and $k$ (generated by epistasis among those loci) tends to be stronger on hap-

$$
+2 \sum_{i, j<k} a_{j, k}\left(\tilde{D}_{i j, k}+\tilde{D}_{i k, j}\right)+2 \sum_{i, j, k} a_{j k, j}\left(\tilde{D}_{i j k, j}+\tilde{D}_{i j, j k}\right)
$$

hat appear 
lotypes that also carry an allele increasing the selfing rate at locus $i$. Indeed, the magnitude of $D_{j k}$ depends on the relative forces of selection generating $D_{j k}$ and recombination breaking it, and selfing affects both processes: by increasing homozygosity, selfing reduces the effect of recombination (e.g., Nordborg, 1997), but it also increases "effective" epistasis, given that when a beneficial combination of alleles is present on one haplotype of an individual, it also tends to be present on the other haplotype due to homozygosity, enhancing the effect of fitness differences between haplotypes.

An expression for $\tilde{D}_{i j k}$ at QLE is given in Supplementary File S4, showing that $\tilde{D}_{i j k}$ is generated by all epistatic components $\left(a_{j k}, a_{j, k}, a_{j k, j}, a_{j k, k}\right.$ and $\left.a_{j k, j k}\right)$. In the case of uniformly deleterious alleles with fixed epistasis (equation 9), one obtains:

$$
\Delta_{\mathrm{LD}} \bar{\sigma} \approx \mathcal{E}\left[\frac{e_{\mathrm{axa}}\left(2+\rho_{j k}{ }^{2}\right)+e_{\mathrm{axd}}+\left(e_{\mathrm{axd}}+\frac{1}{2} e_{\mathrm{dxd}}\right)\left[1-2 \rho_{j k}\left(1-\rho_{j k}\right)\right]}{\rho_{i j k}-\left(1-\rho_{i j k}\right)\left(a_{j}+a_{k}+e_{\mathrm{axa}}\right)}\right] e_{\mathrm{axa}} n_{\mathrm{d}}{ }^{2} \frac{V_{\sigma}}{2}
$$

where $\mathcal{E}$ is the average over all triplets of loci $i, j$ and $k, \rho_{i j k}$ is the probability that at least one recombination event occurs between the three loci $i, j$ and $k$ during meiosis (note that the denominator is approximately $\rho_{i j k}$ when recombination rates are large relative to selection coefficients), and where $n_{\mathrm{d}}$ is the mean number of deleterious alleles per haploid genome. Assuming free recombination among all loci $\left(\rho_{j k}=1 / 2\right.$, $\left.\rho_{i j k}=3 / 4\right)$, equation 43 simplifies to:

$$
\Delta_{\mathrm{LD}} \bar{\sigma} \approx \frac{e_{\mathrm{axa}}}{6}\left(9 e_{\mathrm{axa}}+6 e_{\mathrm{axd}}+e_{\mathrm{dxd}}\right) n_{\mathrm{d}}^{2} V_{\sigma}
$$

“44 Using Charlesworth et al.'s (1991) fitness function, equation 44 yields:

$$
\Delta_{\mathrm{LD}} \bar{\sigma} \approx\left[\beta h(1+h) n_{\mathrm{d}}\right]^{2} \frac{V_{\sigma}}{6}
$$

Finally, under stabilizing selection acting on quantitative traits (and assuming that 
recombination rates are not too small), one obtains:

$$
\Delta_{\mathrm{LD}} \bar{\sigma} \approx \mathcal{E}\left[\frac{2+\rho_{j k}^{2}}{\rho_{i j k}}\right] \frac{2 U^{2}}{n} V_{\sigma}
$$

467

As in the previous section, the term $\Delta_{\text {purge }} \bar{\sigma}$ equals $2 \sum_{i, j} a_{j} \tilde{D}_{i j}$ under random mating and represents indirect selection for selfing due to the fact that selfing increases the efficiency of selection against deleterious alleles. At QLE and to the first order in $a_{\mathbb{U}, \mathbb{V}}$ coefficients, the linkage disequilibrium $\tilde{D}_{i j}$ is given by (see Supplementary File S4 for derivation):

$$
\begin{aligned}
\tilde{D}_{i j} \approx & \frac{1}{2} \frac{\tilde{D}_{i i} p_{j} q_{j}}{\rho_{i j}-a_{j}\left(1-2 p_{j}\right)\left(1-\rho_{i j}\right)}\left[a_{j}+a_{j, j}\left(1-2 p_{j}\right)\right. \\
& \left.+\sum_{k}\left[a_{j k, k}+\left[a_{j k, k}+a_{j k, j k}\left(1-2 p_{j}\right)\right]\left[1-2 \rho_{j k}\left(1-\rho_{j k}\right)\right]\right] p_{k} q_{k}\right] .
\end{aligned}
$$

The term on the first line of equation 47 is the same as in equation 33, representing the fact that increased homozygosity at locus $j$ improves the efficiency of selection acting at this locus. Note that epistatic interactions may affect this term (in particular when the average epistasis between selected loci differs from zero) through the selection coefficients $a_{j}$ and $a_{j, j}$ as well as equilibrium allele frequencies $p_{j}$. The term in the second line of equation 47 shows that negative additive-by-dominance or dominanceby-dominance epistasis between deleterious alleles increase the benefit of selfing, by increasing the efficiency of selection against deleterious alleles in homozygous individuals. In the case of unconditionally deleterious alleles with fixed epistasis, one obtains 
(to the first order in epistatic coefficients):

$$
\begin{aligned}
\Delta_{\text {purge }} \bar{\sigma} \approx \mathcal{E} & {\left[\frac{h\left[s(1-h)-3 e_{\text {axd }} n_{\mathrm{d}}-\left[1-2 \rho_{j k}\left(1-\rho_{j k}\right)\right]\left(e_{\mathrm{axd}}+e_{\mathrm{dxd}}\right) n_{\mathrm{d}}\right]-2 e_{\mathrm{axa}} n_{\mathrm{d}}}{\rho_{i j}-\left(1-\rho_{i j}\right) a_{j}}\right] } \\
& \times s n_{\mathrm{d}} \frac{V_{\sigma}}{2} .
\end{aligned}
$$

Under free recombination, this simplifies to:

$$
\Delta_{\text {purge }} \bar{\sigma} \approx\left[h\left[2 s(1-h)-\left(7 e_{\mathrm{axd}}+e_{\mathrm{dxd}}\right) n_{\mathrm{d}}\right]-4 e_{\mathrm{axa}} n_{\mathrm{d}}\right] s n_{\mathrm{d}} \frac{V_{\sigma}}{4}
$$

Under Gaussian stabilizing selection, the coefficients $a_{j k, j}$ and $a_{j k, j k}$ are small relative to the other selection coefficients (as shown in Supplementary File S1), and the term on the second line of equation 47 may thus be neglected (in which case $\Delta_{\text {purge }} \bar{\sigma}$ is still given by equation 38). With a flatter fitness peak (equation 15 with $Q>2$ ), using the expressions for $a_{j k, j}$ and $a_{j k, j k}$ given by equations A54 and A55 in Supplementary File S1 yields:

$$
\Delta_{\text {purge }} \bar{\sigma} \approx \frac{U^{2}}{\rho_{\mathrm{h}, \sigma z}}\left[3+\frac{7(Q-2)}{4}\right]\left(\frac{4 U}{Q \bar{\varsigma}}\right)^{-\frac{2}{Q}} V_{\sigma}
$$

where the term in $Q-2$ between brackets corresponds to the term on the second line of equation 47 (effects of additive-by-dominance and dominance-by-dominance epistasis).

Figure 3 shows the parameter space (in the $\kappa-\delta^{\prime}$ plane) in which an initially outcrossing population $(\bar{\sigma}=0)$ evolves towards selfing, in the case of uniformly deleterious alleles (fixed epistasis, equation 9). Note that when selfing increased in the simulations (green dots), we always observed that the population evolved towards selfing rates close to 1 . Figures $3 \mathrm{~A}-\mathrm{C}$ show that negative $e_{\mathrm{axd}}$ or $e_{\mathrm{dxd}}$ (the other epistatic components being set to zero) slightly increase the parameter range under which selfing evolves: in particular, selfing can invade for values of inbreeding depression $\delta^{\prime}$ slightly higher than 0.5 in the absence of pollen discounting $(\kappa=0)$. Epistasis has stronger 
effects when negative $e_{\mathrm{axd}}$ and/or $e_{\mathrm{dxd}}$ are combined with negative $e_{\mathrm{axa}}$, as shown by Figures 3D-F (we did not test the effect of negative $e_{\text {axa }}$ alone, as $\delta^{\prime}$ is greatly reduced in this case unless $e_{\text {axa }}$ is extremely weak). The QLE model (dashed and solid curves) correctly predicts the maximum inbreeding depression $\delta^{\prime}$ for selfing to evolve, as long as this maximum is not too large: high values of $\delta^{\prime}$ indeed imply high values of $U$, for which the QLE model overestimates the strength of indirect effects (in particular, the model predicts that selfing may evolve under high depression, above the upper parts of the curves in Figures 3D-F, but this was never observed in the simulations). In all cases shown in Figure 3, the increased parameter range under which selfing can evolve is predicted to be mostly due to the effect of negative epistasis on $\Delta_{\text {purge }} \bar{\sigma}$, the effect of $\Delta_{\mathrm{LD}} \bar{\sigma}$ remaining negligible. Finally, one can note that the maximum $\delta^{\prime}$ for selfing to evolve is lower with $e_{\mathrm{axa}}=-0.005, e_{\mathrm{axd}}=e_{\mathrm{dxd}}=-0.01$ (Figure 3E) than with $e_{\mathrm{axa}}=-0.005, e_{\mathrm{axd}}=-0.01, e_{\mathrm{dxd}}=0$ (Figure $\left.3 \mathrm{D}\right)$. This is due to the fact that negative $e_{\mathrm{axd}}$ and $e_{\mathrm{dxd}}$ have two opposite effects: they increase the effect of selection against homozygous mutations (which increases $\Delta_{\text {purge }} \bar{\sigma}$ ), but they also increase the strength of inbreeding depression for a given mutation rate $U$ (see Figure 1), decreasing the mean number of deleterious alleles per haplotype $n_{\mathrm{d}}$ associated with a given value of $\delta^{\prime}$ (which decreases $\left.\Delta_{\text {purge }} \bar{\sigma}\right)$.

Supplementary Figure S1 shows the effect of the size of mutational steps at the selfing modifier locus, in the absence of epistasis (corresponding to Figure 3A), and with all three components of epistasis being negative (corresponding to Figure $3 \mathrm{E})$. Increasing the size of mutational steps has more effect in the presence of negative epistasis, since negative epistasis increases the purging advantage of alleles coding for more selfing $\left(\Delta_{\text {purge }} \bar{\sigma}\right)$, whose effect becomes stronger relative to $\Delta_{\text {auto }} \bar{\sigma}$ and $\Delta_{\text {depr }} \bar{\sigma}$ 
when modifier alleles have larger effects (as previously shown in Figure 2).

Figure 4 shows the results obtained under Gaussian stabilizing selection (equation 14) acting on different numbers of traits $n$, keeping the mean deleterious effect of mutations $\bar{\varsigma}$ constant. Under stabilizing selection, inbreeding depression reaches an upper limit as the mutation rate $U$ increases (this upper limit being lower for lower values of $n$ ), explaining why high values of $\delta^{\prime}$ could not be explored in Figure 4. Again, epistasis increases the parameter range under which selfing can invade (the effect of epistasis being stronger when the number of selected traits $n$ is lower), and the QLE model yields correct predictions as long as inbreeding depression (and thus $U$ ) is not too large. In contrast with the fixed epistasis model discussed above, the model predicts that $\Delta_{\text {purge }} \bar{\sigma}$ stays negligible, the difference between the dotted and solid/dashed curves in Figure 4 being mostly due to $\Delta_{\mathrm{LD}} \bar{\sigma}$ : selfers thus benefit from the fact that they can maintain beneficial combinations of alleles (mutations with compensatory effects) at different loci. Interestingly, for $n=5$ and sufficiently high rates of pollen discounting $\kappa$, selfing can invade if inbreeding depression is lower than a given threshold, or is very high. The latter case corresponds to a situation where polymorphism is important (high $U$ ) and where large numbers of compensatory combinations of alleles are possible. Although the model predicts that the same phenomenon should occur for higher values of $n$, it was not observed in simulations with $n=15$ and $n=30$, except for $n=15$ and $\kappa=0.4$. However, Supplementary Figures S2 and S3 show that the evolution of selfing above a threshold value of $\delta^{\prime}$ occurs more frequently when the fitness peak is flatter $(Q>2)$, and when mutations affecting the selfing rate have larger effects.

Finally, Figure 5 provides additional results on the effect of the number of se- 
lected traits $n$, for fixed values of the overall mutation rate $U$. Inbreeding depression is little affected by epistatic interactions when $n$ is large, while low values of $n$ tend to decrease inbreeding depression, explaining the shapes of the dotted curves showing the maximum level of pollen discounting for selfing to spread, when only taking into account the effects of the automatic advantage and inbreeding depression. The difference between the dotted and solid/dashed curves shows the additional effect of linkage disequilibria generated by epistasis $\left(\Delta_{\mathrm{LD}} \bar{\sigma}\right)$, whose relative importance increases as the number of traits $n$ decreases, and as the mutation rate $U$ increases. Because $U$ stays moderate $(U=0.2$ or 0.5$)$, the analytical model provides accurate predictions of the parameter range in which selfing is favored.

\section{DISCUSSION}

The automatic transmission advantage associated with selfing and inbreeding depression are the two most commonly discussed genetic mechanisms affecting the evolution of self-fertilization. When these are the only forces at play, a selfing mutant arising in an outcrossing population is expected to increase in frequency as long as inbreeding depression is weaker than the automatic advantage, whose magnitude depends on the level of pollen discounting (Lande and Schemske, 1985; Holsinger et al., 1984). However, because selfers also tend to carry better purged genomes due to their increased homozygosity, several models showed that selfing mutants may invade under wider conditions than those predicted solely based on these two aforementioned forces (Charlesworth et al., 1990; Uyenoyama and Waller, 1991; Epinat and Lenormand, 2009; Porcher and Lande, 2005b; Gervais et al., 2014). Our analytical and simulation results 
confirm that the advantage procured through purging increases with the strength of selection against deleterious alleles and with the degree of linkage within the genome. The simulation results also indicate that the verbal prediction, according to which mutations causing complete selfing may invade a population independently of its level of inbreeding depression (Lande and Schemske, 1985, p. 33), only holds when deleterious alleles have strong fitness effects, so that purging occurs rapidly (Figure 2D).

Whether purging efficiency should significantly contribute to the spread of selfing mutants depends on the genetic architecture of inbreeding depression. To date, experimental data point to a small contribution of strongly deleterious alleles to inbreeding depression: for example, Baldwin and Schoen (2019) recently showed that in the self-incompatible species Leavenworthia alabamica, inbreeding depression is not affected by three generations of enforced selfing (which should have lead to the elimination of deleterious alleles with strong fitness effects). Previous experiments on different plant species also indicate that inbreeding depression is probably generated mostly by weakly deleterious alleles (Dudash et al., 1997; Willis, 1999; Carr and Dudash, 2003; Charlesworth and Willis, 2009). Data on the additive variance in fitness within populations are also informative regarding the possible effect of purging: indeed, using our general expression for fitness (equation 8) and neglecting linkage disequilibria, one can show that the additive component of the variance in fitness in a randomly mating population (more precisely, the variance in $W / \bar{W}$ ) is given by the sum over selected loci of $2 a_{j}{ }^{2} p_{j} q_{j}$ (see also eq. A3b in Charlesworth and Barton, 1996), a term which also appears in the effect of purging on the strength of selection for selfing (equation 35). Although estimates of the additive variance in fitness in wild populations remain scarce, the few estimates of the "evolvability" parameter (corresponding 
to the additive component of the variance in $W / \bar{W}$ ) available from plant species are small, of the order of a few percents (Hendry et al., 2018). Note that strictly, the effect of purging on the strength of selection for selfing is proportional to the quantity $\sum_{j} a_{j}\left[a_{j}+a_{j, j}\left(1-2 p_{j}\right)\right] p_{j} q_{j}$ (equation 35), which may be larger than $\sum_{j} a_{j}^{2} p_{j} q_{j}$ (for example, in the case of deleterious alleles with fixed $s$ and $h$, the first quantity is approximately $s(1-h) U$ and the second $s h U)$. However, the small values of the available estimates of $\sum_{j} a_{j}{ }^{2} p_{j} q_{j}$, together with the experimental evidence mentioned above on the genetics of inbreeding depression, indicate that selfing mutants probably do not benefit greatly from purging. Nevertheless, it remains possible that the strength of selection against deleterious alleles $\left(a_{j}\right)$ increases in harsher environments (Cheptou et al., 2000; Agrawal and Whitlock, 2010), leading to stronger purging effects in such environments.

The effects of epistasis between deleterious alleles on inbreeding depression and on the evolution of mating systems have been little explored (but see Charlesworth et al., 1991). In this paper, we derived general expressions for the effect of epistasis between pairs of loci on inbreeding depression and on the strength of selection for selfing, that can be applied to more specific models. Our results show that different components of epistasis have different effects on inbreeding depression: in particular, while negative additive-by-additive epistasis tends to lower inbreeding depression by reducing the frequency of deleterious alleles, negative additive-by-dominance and dominance-bydominance epistasis increase inbreeding depression by lowering the fitness of homozygous offspring. Very little is known on the average sign and relative magnitude of these different forms of epistasis. In principle, the overall sign of dominance-by-dominance effects can be deduced from the shape of the relation between the inbreeding coefficient 
of individuals $(F)$ and their fitness (Crow and Kimura, 1970, p. 80), an accelerating decline in fitness as $F$ increases indicating negative $e_{\mathrm{dxd}}$. The relation between $F$ and fitness-related traits was measured in several plant species; the results often showed little departure from linearity (e.g., Willis, 1993; Kelly, 2005), but the experimental protocols used may have generated biases against finding negative $e_{\mathrm{dxd}}$ (Falconer and Mackay, 1996; Lynch and Walsh, 1998; Sharp and Agrawal, 2016). Most empirical distributions of epistasis between pairs of mutations affecting fitness have been obtained from viruses, bacteria and unicellular eukaryotes (e.g., Martin et al., 2007; Kouyos et al., 2007; de Visser and Elena, 2007). While no clear conclusion emerges regarding the average coefficient of epistasis (some studies find that it is negative, other positive and other close to zero), a general observation is that epistasis is quite variable across pairs of loci. This variance of epistasis may slightly increase inbreeding depression when it remains small (by reducing the efficiency of selection against deleterious alleles, Phillips et al., 2000; Abu Awad and Roze, 2018), or decrease inbreeding depression when it is larger and/or effective recombination is sufficiently weak, so that selfing can maintain beneficial multilocus genotypes (Lande and Porcher, 2015; Abu Awad and Roze, 2018). Besides this "short-term" effect on inbreeding depression, the variance of epistasis also favors selfing through the progressive buildup of linkage disequilibria that increase mean fitness (associations between alleles with compensatory effects at different loci). Interestingly, this effect may allow selfers to spread above a threshold value of the rate of mutation on traits under stabilizing selection (Figures 4, S3). Is the variance of epistasis typically large enough, so that this benefit of maintaining beneficial combinations of alleles may significantly help selfing mutants to spread? Answering this question is difficult without better knowl- 
edge on the importance of epistatic interactions on fitness in natural environments.

Nevertheless, some insights can be gained from our analytical results: for example, neglecting additive-by-dominance and dominance-by-dominance effects, equations 42 and D7 indicate that the effect of linkage disequilibria on the strength of selection for selfing should scale with the sum over pairs of selected loci of $a_{j k}^{2} p_{j} q_{j} p_{k} q_{k}$, which also corresponds to the epistatic component of the variance in fitness in randomly mating populations. Although estimates of epistatic components of variance remain scarce, they are typically not larger than additive components (e.g., Hill et al., 2008), suggesting that the benefit of maintaining beneficial multilocus genotypes may be generally limited (given that the additive variance in fitness seems typically small, as discussed previously).

A mixed mating system was never stably maintained in our simulations: the selfing rate always evolved towards a value either close to zero or one. Using a deterministic model, Charlesworth et al. (1991) showed that in the presence of negative epistasis between deleterious alleles, and when outcrossing is not stable, a selfing rate slightly below one corresponds to the evolutionarily stable strategy (ESS). This can be understood from the fact that negative epistasis favors non-zero rates of recombination (e.g., Barton, 1995), while recombination becomes ineffective under complete selfing. Similarly, Kamran-Disfani and Agrawal (2014) showed that selfing rates slightly below one are selectively favored over complete selfing in finite populations, when deleterious alleles occur at multiple loci: again, this probably results from selection for recombination, generated by Hill-Robertson effects between selected loci (e.g., Barton and Otto, 2005). Similar effects must have occurred in our simulations, although we did not check that selfing rates slightly below one resulted from selection to maintain low 
rates of outcrossing, rather than from the constant input of mutations at selfing modifier loci (this could be done by comparing the probabilities of fixation of alleles coding for different selfing rates, as in Kamran-Disfani and Agrawal, 2014). It is possible that conditions (for example, under directional selection acting on quantitative traits) than under the stable conditions considered in the present paper; this represents an interesting avenue for future research.

675

Acknowledgements: We thank the bioinformatics and computing service of Roscoff's Biological Station (Abims platform) for computing time. This work was supported by the French Agence Nationale de la Recherche (project SEAD, ANR-13-ADAP-0011 and project SexChange, ANR-14-CE02-0001). Diala Abu Awad wad partly funded by the TUM University Foundation Fellowship. 
Abu Awad, D. and D. Roze. 2018. Effects of partial selfing on the equilibrium genetic variance, mutation load, and inbreeding depression under stabilizing selection. Evolution 72:751-769.

Agrawal, A. F. and M. C. Whitlock. 2010. Environmental duress and epistasis: how does stress affect the strength of selection on new mutations? Trends Ecol. Evol. 25:450-458.

Baldwin, S. J. and D. J. Schoen. 2019. Inbreeding depression is difficult to purge in self-incompatible populations of Leavenworthia alabamica. New Phytol. doi: 10.1111/nph.15963.

Barrett, S. C. H., R. Arunkumar, and S. I. Wright. 2014. The demography and population genomics of evolutionary transitions to self-fertilization in plants. Phil. Trans. Roy. Soc. (Lond.) B 369:20130344.

Barton, N. H. 1995. A general model for the evolution of recombination. Genet. Res. 65:123-144.

Barton, N. H. and S. P. Otto. 2005. Evolution of recombination due to random drift. Genetics 169:2353-2370.

Barton, N. H. and M. Turelli. 1991. Natural and sexual selection on many loci. Genetics $127: 229-255$.

Busch, J. W. and L. F. Delph. 2012. The relative importance of reproductive assurance 
and automatic selection as hypotheses for the evolution of self-fertilization. Ann. Bot. 109:553-562.

Carr, D. E. and M. R. Dudash. 2003. Recent approaches into the genetic basis of inbreeding depression in plants. Phil. Trans. Roy. Soc. (Lond.) B 358:1071-1084.

Charlesworth, B. 1980. The cost of sex in relation to the mating system. J. Theor. Biol. 84:655-671.

Charlesworth, B. and N. H. Barton. 1996. Recombination load associated with selection for increased recombination. Genet. Res. 67:27-41.

Charlesworth, B., M. T. Morgan, and B. Charlesworth. 1990. Inbreeding depression, genetic load, and the evolution of outcrossing rates in a multilocus system with no linkage. Evolution 44:1469-1489.

Charlesworth, B., M. T. Morgan, and D. Charlesworth. 1991. Multilocus models of inbreeding depression with synergistic selection and partial self-fertilization. Genet. Res. 57:177-194.

Charlesworth, D. and B. Charlesworth. 1987. Inbreeding depression and its evolutionary consequences. Ann. Rev. Ecol. Syst. 18:237-268.

Charlesworth, D. and J. H. Willis. 2009. The genetics of inbreeding depression. Nat. Rev. Genet. 10:783-796.

Cheptou, P. O., E. Imbert, J. Lepart, and J. Escarre. 2000. Effects of competition on lifetime estimates of inbreeding depression in the outcrossing plant Crepis sancta (Asteraceae). J. Evol. Biol. 13:522-531. 
Crow, J. F. and M. Kimura. 1970. An Introduction to Population Genetics Theory. Harper and Row, New York.

Darwin, C. 1876. The effects of cross- and self-fertilization in the vegetable kingdom. John Murray, London.

de Visser, J. A. G. M. and S. F. Elena. 2007. The evolution of sex: empirical insights into the roles of epistasis and drift. Nat. Rev. Genet. 8:139-149.

Dudash, M. R., D. E. Carr, and C. B. Fenster. 1997. Five generations of enforced selfing and outcrossing in Mimulus guttatus: inbreeding depression variation at the population and family level. Evolution 51:64-65.

Epinat, G. and T. Lenormand. 2009. The evolution of assortative mating and selfing with in- and outbreeding depression. Evolution 63:2047-2060.

Falconer, D. S. and T. F. C. Mackay. 1996. Introduction to Quantitative Genetics. Addison Wesley Longman, Harlow.

Fisher, R. 1941. Average excess and average effect of a gene substitution. Ann. Eugen. 11:53-63.

Gervais, C., D. Abu Awad, D. Roze, V. Castric, and S. Billiard. 2014. Genetic architecture of inbreeding depression and the maintenance of gametophytic selfincompatibility. Evolution 68:3317-3324.

Gros, P.-A., H. Le Nagard, and O. Tenaillon. 2009. The evolution of epistasis and its links with genetic robustness, complexity and drift in a phenotypic model of adaptation. Genetics 182:277-293. 
Hendry, A. P., D. J. Schoen, M. E. Wolak, and J. M. Reid. 2018. The contemporary evolution of fitness. Ann. Rev. Ecol. Evol. Syst. 49:457-476.

Hill, W. G., M. E. Goddard, and P. M. Visscher. 2008. Data and theory point to mainly additive genetic variance for complex traits. PLoS Genetics 4:e1000008.

Holsinger, K. E. 1991. Mass-action models of plant mating systems: the evolutionary stability of mixed mating systems. Am. Nat. 138:606-622.

Holsinger, K. E., M. W. Feldman, and F. B. Christiansen. 1984. The evolution of self-fertilization in plants: a population genetic model. Am. Nat. 124:446-453.

Igic, B. and J. W. Busch. 2013. Is self-fertilization an evolutionary dead end? New Phytol. 198:386-397.

Jarne, P. and J. R. Auld. 2006. Animals mix it up too: the distribution of selffertilization among hermaphroditic animals. Evolution 60:1816-1824.

Kamran-Disfani, A. and A. F. Agrawal. 2014. Selfing, adaptation and background selection in finite populations. J. Evol. Biol. 27:1360-1371.

Kelly, J. K. 2005. Epistasis in monkeyflowers. Genetics 171:1917-1931.

Kirkpatrick, M., T. Johnson, and N. H. Barton. 2002. General models of multilocus evolution. Genetics 161:1727-1750.

Kouyos, R. D., O. K. Silander, and S. Bonhoeffer. 2007. Epistasis between deleterious mutations and the evolution of recombination. Trends Ecol. Evol. 22:308-315. 
Lande, R. and E. Porcher. 2015. Maintenance of quantitative genetic variance under partial self-fertilization, with implications for the evolution of selfing. Genetics 200:891-906.

Lande, R. and D. W. Schemske. 1985. The evolution of self-fertilization and inbreeding depression in plants. I. Genetic models. Evolution 39:24-40.

Lynch, M. and J. B. Walsh. 1998. Genetics and Analysis of Quantitative Traits. Sinauer Associates, Sunderland, MA.

Manna, F., G. Martin, and T. Lenormand. 2011. Fitness landscapes: an alternative theory for the dominance of mutation. Genetics 189:923-937.

Martin, G., S. F. Elena, and T. Lenormand. 2007. Distributions of epistasis in microbes fit predictions from a fitness landscape model. Nat. Genet. 39:555-560.

Martin, G. and T. Lenormand. 2006a. The fitness effect of mutations across environments: a survey in light of fitness landscape models. Evolution 60:2413-2427.

— 2006b. A general multivariate extension of Fisher's geometrical model and the distribution of mutation fitness effects across species. Evolution 60:893-907.

Nagylaki, T. 1976. A model for the evolution of self-fertilization and vegetative reproduction. J. Theor. Biol. 58:55-58.

- 1993. The evolution of multilocus systems under weak selection. Genetics 134:627-647.

Nordborg, M. 1997. Structured coalescent processes on different time scales. Genetics $146: 1501-1514$. 
Phillips, P. C., S. P. Otto, and M. C. Whitlock. 2000. Beyond the average: the evolutionary importance of gene interactions and variability of epistatic effects. Pp. 20-38 in J. B. Wolf, E. D. Brodie, and M. J. Wade, eds. Epistasis and the Evolutionary Process. Oxford University Press, New York.

Porcher, E. and R. Lande. 2005a. The evolution of self-fertilization and inbreeding depression under pollen discounting and pollen limitation. J. Evol. Biol. 18:497508.

— 2005b. Loss of gametophytic self-incompatibility with evolution of inbreeding depression. Evolution 59:46-60.

Roze, D. and A. Blanckaert. 2014. Epistasis, pleiotropy and the mutation load in sexual and asexual populations. Evolution 68:137-149.

Sharp, N. P. and A. F. Agrawal. 2016. The decline in fitness with inbreeding: evidence for negative dominance-by-dominance epistasis in Drosophila melanogaster. J. Evol. Biol. 29:857-864.

Stebbins, G. L. 1957. Self fertilization and population variability in higher plants. Am. Nat. 91:337-354.

Stone, J. L., E. J. Van Wyk, and J. R. Hale. 2014. Transmission advantage favors selfing allele in experimental populations of self-incompatible Witheringia solanacea (Solanaceae). Evolution 68:1845-1855.

Tenaillon, O., O. K. Silander, J.-P. Uzan, and L. Chao. 2007. Quantifying organismal complexity using a population genetic approach. PLoS One 2:e217. 
804

805

806

807

808

809

810

811

812

Uyenoyama, M. K. and D. M. Waller. 1991. Coevolution of self-fertilization and inbreeding depression. I. Mutation-selection balance at one and two loci. Theor. Popul. Biol. 40:14-46.

Weir, B. S. and C. C. Cockerham. 1973. Mixed self and random mating at two loci. Genet. Res. 21:247-262.

Willis, J. H. 1993. Effects of different levels of inbreeding on fitness components in Mimulus guttatus. Evolution 47:864-876.

1 1999. The role of genes of large effect on inbreeding depression in Mimulus guttatus. Evolution 53:1678-1691. 
Table 1: Parameters and variables of the model.

\begin{tabular}{|c|c|}
\hline$\sigma$ & Selfing rate \\
\hline $\bar{\sigma}, V_{\sigma}$ & Mean and variance in the selfing rate in the population \\
\hline$\kappa$ & Rate of pollen discounting \\
\hline$\ell_{\sigma}$ & Number of loci affecting the selfing rate \\
\hline$W, \bar{W}$ & Fitness of an individual, and average fitness \\
\hline$\ell$ & Number of loci affecting fitness \\
\hline$U$ & Overall (haploid) mutation rate at loci affecting fitness \\
\hline$p_{j}, q_{j}$ & Frequencies of alleles 1 and 0 at loci affecting fitness \\
\hline$\ell$ & Number of loci affecting selected traits \\
\hline$n_{\mathrm{d}}$ & Mean number of deleterious alleles per haploid genome \\
\hline$s, h$ & Selection and dominance coefficients of deleterious alleles \\
\hline$e_{\text {axa }}, e_{\text {axd }}, e_{\mathrm{dxd}}$ & $\begin{array}{l}\text { Additive-by-additive, additive-by-dominance and } \\
\text { dominance-by-dominance epistasis between deleterious alleles }\end{array}$ \\
\hline$\beta$ & $\begin{array}{l}\text { Strength of synergistic epistasis in Charlesworth et al.'s (1991) } \\
\text { model }\end{array}$ \\
\hline$n$ & Number of quantitative traits under stabilizing selection \\
\hline$V_{\mathrm{s}}$ & Strength of stabilizing selection \\
\hline$r_{\alpha j}$ & Effect of allele 1 at locus $j$ on trait $\alpha$ \\
\hline
\end{tabular}




\begin{tabular}{|c|c|}
\hline$a^{2}$ & $\begin{array}{l}\text { Variance of mutational effects on traits under stabilizing } \\
\text { selection }\end{array}$ \\
\hline$Q$ & Shape of the fitness peak (equation 15) \\
\hline$a_{\mathbb{U}, \mathbb{V}}$ & $\begin{array}{l}\text { Effect of selection on the sets } \mathbb{U} \text { and } \mathbb{V} \text { of loci present on the } \\
\text { maternally and paternally inherited haplotypes of an individual } \\
\text { (equation 8) }\end{array}$ \\
\hline$D_{\mathbb{U}, \mathbb{V}}$ & $\begin{array}{l}\text { Genetic association between the sets } \mathbb{U} \text { and } \mathbb{V} \text { of loci present on } \\
\text { the maternally and paternally inherited haplotypes of an } \\
\text { individual (equation } 4 \text { ) }\end{array}$ \\
\hline$\rho_{j k}$ & Recombination rate between loci $j$ and $k$ \\
\hline$U_{\text {self }}$ & Mutation rate at loci affecting the selfing rate \\
\hline$\sigma_{\text {self }}^{2}$ & Variance of mutational effects at loci affecting the selfing rate \\
\hline$\delta$ & Inbreeding depression \\
\hline$\delta^{\prime}$ & Inbreeding depression measured after selection \\
\hline$F$ & Inbreeding coefficient \\
\hline$G_{j k}$ & Identity disequilibrium between loci $j$ and $k$ \\
\hline$G$ & Identity disequilibrium between freely recombining loci \\
\hline
\end{tabular}



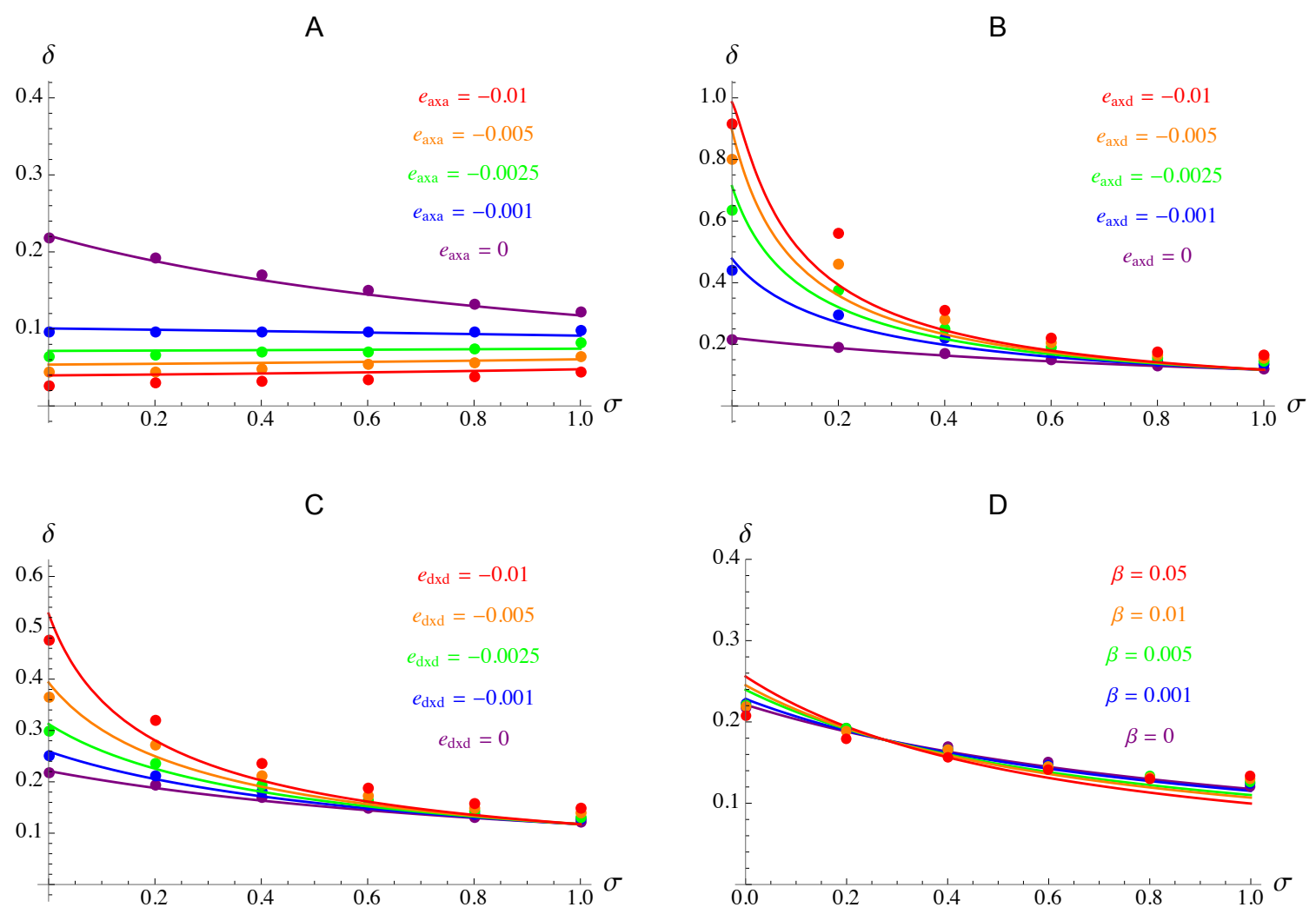

Figure 1. Inbreeding depression $\delta$ as a function of the selfing rate $\sigma$. A-C: effects of the different components of epistasis between deleterious alleles, additive-by-additive $\left(e_{\text {axa }}\right)$, additive-by-dominance $\left(e_{\mathrm{axd}}\right)$ and dominance-by-dominance $\left(e_{\mathrm{dxd}}\right)$ - in each plot, the other two components of epistasis are set to zero. D: results obtained using between deleterious alleles (slightly modified as explained in Supplementary File S1). Dots correspond to simulation results (error bars are smaller than the size of symbols), and curves to analytical predictions from equations 23 and 26. Parameter values: $U=0.25, s=0.05, h=0.25$. In the simulations $N=20,000$ (population size) and $R=20$ (genome map length); simulations lasted $10^{5}$ generations and inbreeding depression was averaged over the last $5 \times 10^{4}$ generations. 

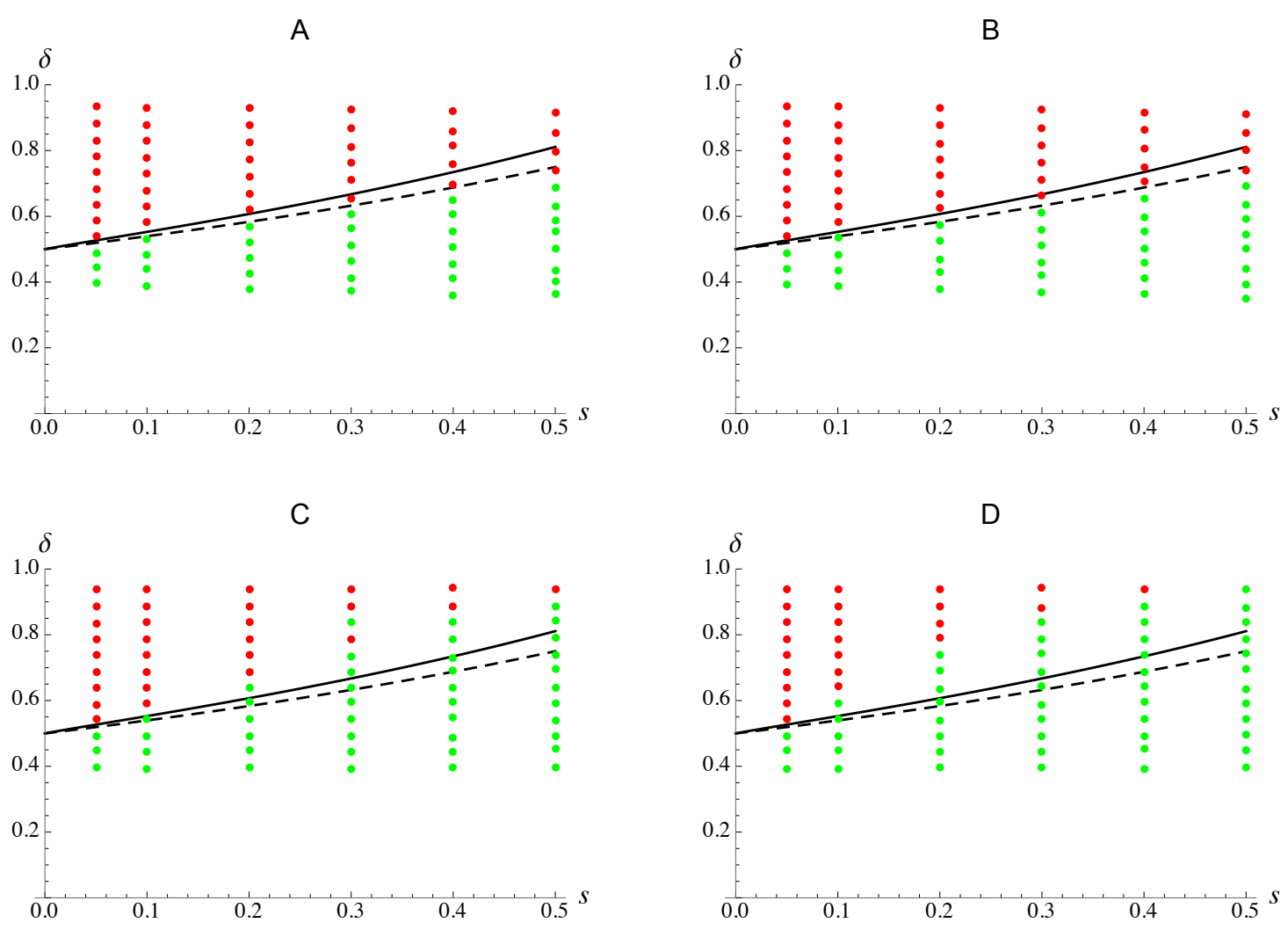

Figure 2. Evolution of selfing in the absence of epistasis. The solid curve shows the maximum value of inbreeding depression $\delta$ for selfing to spread in an initially outcrossing population, as a function of the strength of selection $s$ against deleterious alleles (obtained from equations 34 and 36, after integrating equation 36 over the genetic map), while the dashed curve corresponds to the same prediction in the case of unlinked loci (obtained by setting $\rho_{i j}=1 / 2$ in equation 36). Dots correspond to simulation results (using different values of $U$ for each value of $s$, in order to generate a range of values of $\delta$ ). In the simulations the population evolves under random mating during the first 20,000 generations (inbreeding depression is estimated by averaging over the last 10,000 generations); mutation is then introduced at the selfing modifier locus. A red dot means that the selfing rate stayed below 0.05 during the $2 \times 10^{5}$ 
generations of the simulation, while a green dot means that selfing increased (in which case the population always evolved towards nearly complete selfing). Parameter values: $\kappa=0, h=0.25, R=10$; in the simulations $N=20,000, U_{\text {self }}=0.001$ (mutation rate at the selfing modifier locus). In A, the standard deviation of mutational effects at the modifier locus is set to $\sigma_{\text {self }}=0.01$, while it is set to $\sigma_{\text {self }}=0.03$ in $\mathrm{B}$, and to $\sigma_{\text {self }}=0.3$ 847 in C. In D, only two alleles are possible at the modifier locus, coding for $\sigma=0$ or 1 , 848 respectively. 
A

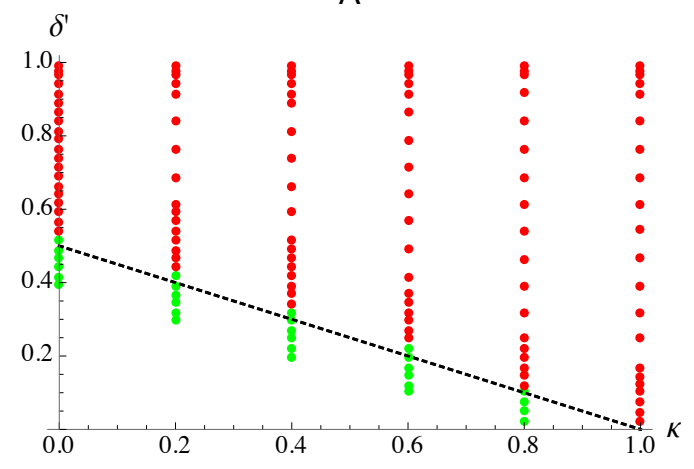

C

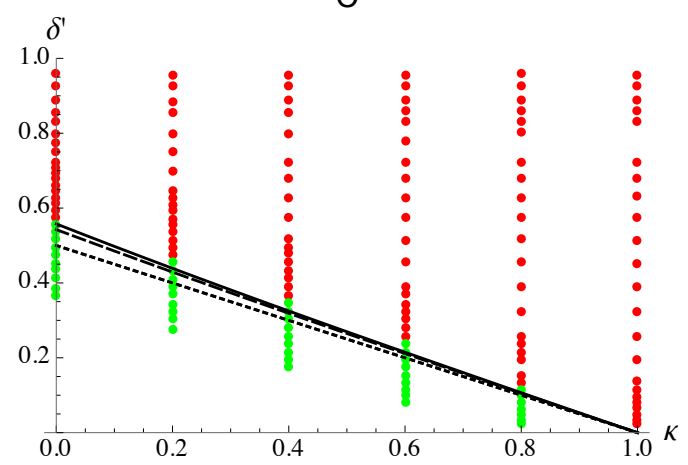

E

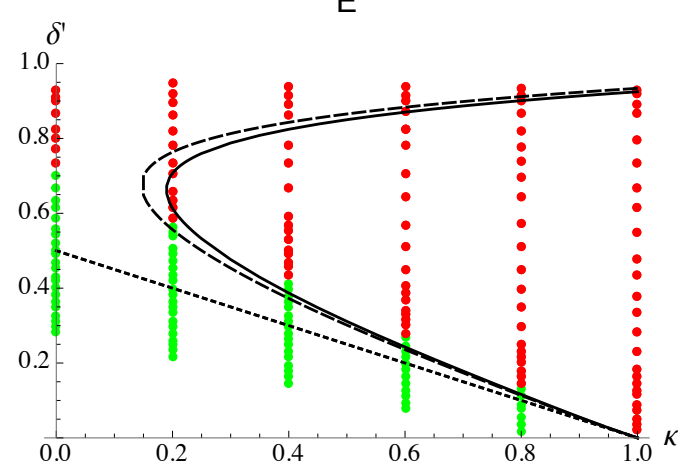

B

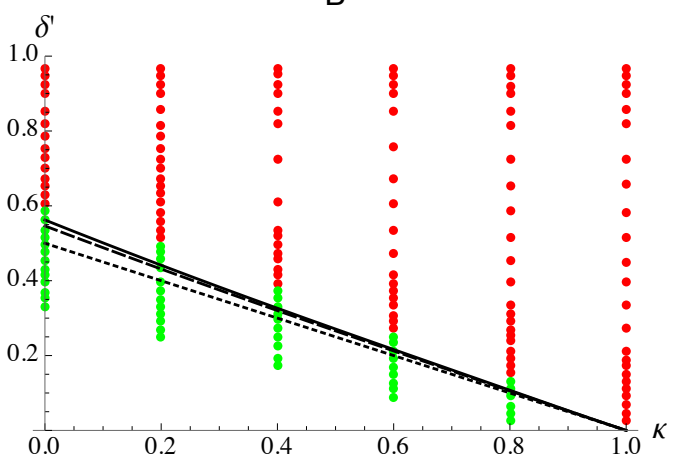

D

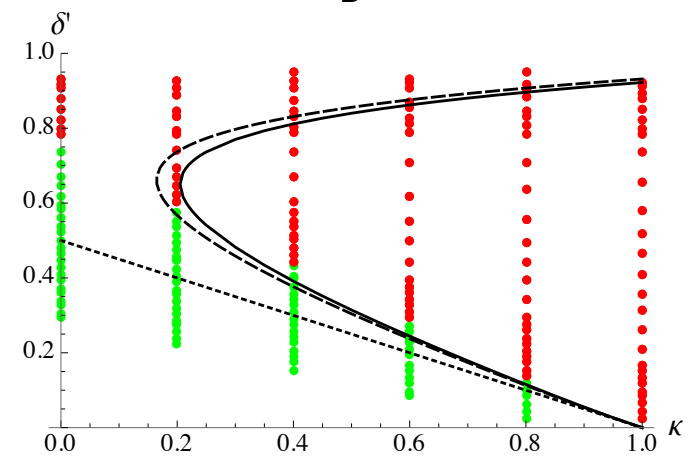

$\mathrm{F}$

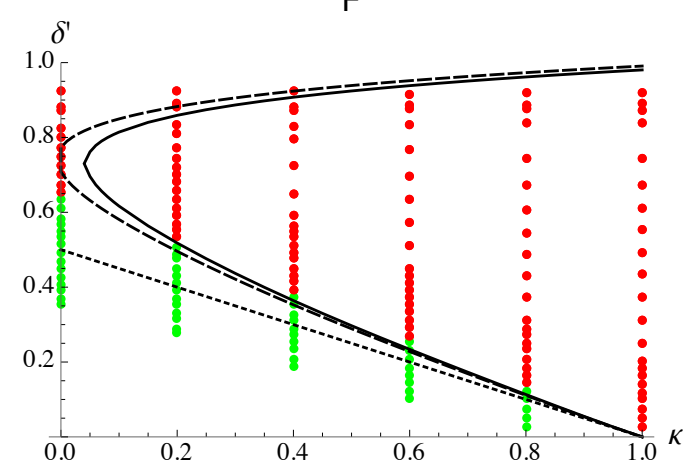

Figure 3. Evolution of selfing with fixed, negative epistasis. The different plots show the maximum value of inbreeding depression $\delta^{\prime}$ (measured after selection) for selfing to spread in an initially outcrossing population, as a function of the rate of pollen discounting $\kappa$. Green and red dots correspond to simulation results and have the same meaning as in Figure $2\left(\delta^{\prime}\right.$ was estimated by averaging over the last 10,000 5 generations of the 20,000 preliminary generations without selfing, simulations lasted 
$2 \times 10^{5}$ generations). The dotted lines correspond to the predicted maximum inbreeding depression for selfing to increase obtained when neglecting $\Delta_{\mathrm{LD}} \bar{\sigma}$ and $\Delta_{\text {purge }} \bar{\sigma}$ (that is, $\left.\delta^{\prime}=(1-\kappa) / 2\right)$, the dashed curves correspond to the prediction obtained using the expressions for $\Delta_{\mathrm{LD}} \bar{\sigma}$ and $\Delta_{\text {purge }} \bar{\sigma}$ under free recombination (equations 44 and 49), while the solid curves correspond to the predictions obtained by integrating equations 43 and 48 over the genetic map (the effect of $\Delta_{\mathrm{LD}} \bar{\sigma}$ is predicted to be negligible relative to the effect of $\Delta_{\text {purge }} \bar{\sigma}$ in all cases). To obtain these predictions, the relation between the mean number of deleterious alleles per haplotype $n_{\mathrm{d}}$ (that appears in equations 43-44 and 48-49) and $\delta^{\prime}$ was obtained from a fit of the simulation results. A: $e_{\text {axa }}=$ $e_{\mathrm{axd}}=e_{\mathrm{dxd}}=0 ; \mathrm{B}: e_{\mathrm{axa}}=e_{\mathrm{dxd}}=0, e_{\mathrm{axd}}=-0.01 ; \mathrm{C}: e_{\mathrm{axa}}=e_{\mathrm{axd}}=0, e_{\mathrm{dxd}}=-0.01$ $\mathrm{D}: e_{\mathrm{axa}}=-0.005, e_{\mathrm{axd}}=-0.01, e_{\mathrm{dxd}}=0 ; \mathrm{E}: e_{\mathrm{axa}}=-0.005, e_{\mathrm{axd}}=e_{\mathrm{dxd}}=-0.01 ; \mathrm{F}:$ Charlesworth et al.'s (1991) model with $\beta=0.05$. Other parameter values: $s=0.05$, $h=0.25, R=20$; in the simulations $N=20,000, U_{\text {self }}=0.001$ (mutation rate at the selfing modifier locus), $\sigma_{\text {self }}=0.03$ (standard deviation of mutational effects at the modifier locus). 

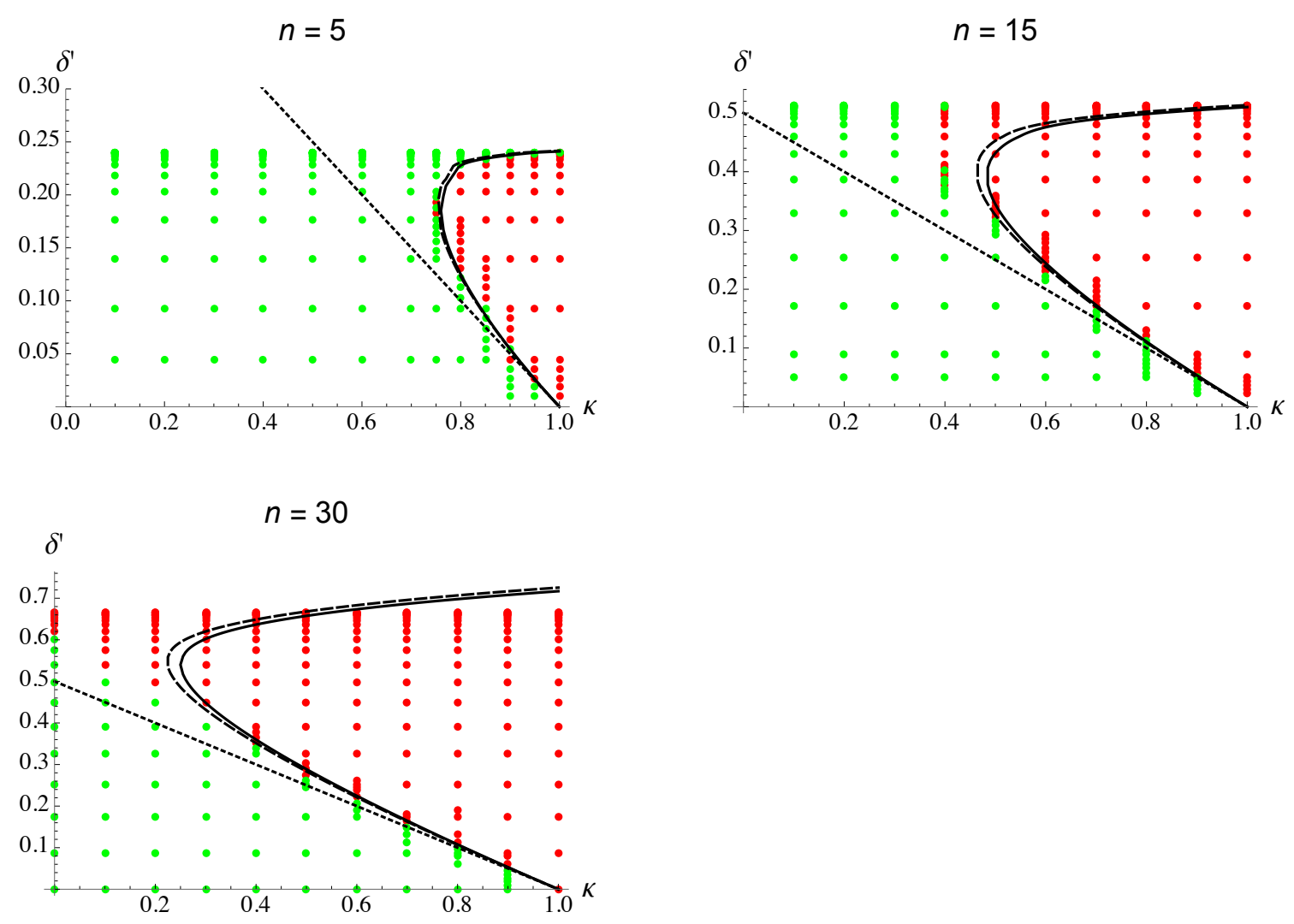

Figure 4. Evolution of self-fertilization under Gaussian stabilizing selection. The three plots show the effects of inbreeding depression $\delta^{\prime}$ (measured after selection) and pollen discounting (parameter $\kappa$ ) on the evolution of self-fertilization, for different numbers of traits under selection $(n=5,15$ and 30$)$. Green and red dots correspond to simulation results and have the same meaning as in Figures 2 and $3\left(\delta^{\prime}\right.$ was estimated without selfing, simulations lasted $5 \times 10^{4}$ generations). The fact that inbreeding depression reaches a plateau as $U$ increases (at lower values of $\delta^{\prime}$ for lower values of $n$ ) sets an upper limit to the values of $\delta^{\prime}$ that can be obtained in the simulations. The dotted lines correspond to the predicted maximum inbreeding depression for selfing to increase obtained when neglecting $\Delta_{\mathrm{LD}} \bar{\sigma}$ and $\Delta_{\text {purge }} \bar{\sigma}$ (that is, $\delta^{\prime}=(1-\kappa) / 2$ ), the 
dashed curves correspond to the prediction obtained using the expression for $\Delta_{\mathrm{LD}} \bar{\sigma}$ under free recombination (that is, $6 U^{2} V_{\sigma} / n$, see equation 46 ), while the solid curves correspond to the predictions obtained by integrating equation 46 over the genetic map (the effect of $\Delta_{\text {purge }} \bar{\sigma}$ is predicted to be negligible relative to the effect of $\Delta_{\mathrm{LD}} \bar{\sigma}$ ).

${ }_{887}$ To obtain these predictions, the relation between $U$ and $\delta^{\prime}$ was obtained from a fit of 888 the simulation results. Other parameter values: $\bar{\varsigma}=0.01, R=20$; in the simulations ${ }_{889} N=5,000, U_{\text {self }}=0.001$ (overall mutation rate at selfing modifier loci), $\sigma_{\text {self }}=0.01$ 890 (standard deviation of mutational effects on selfing). 

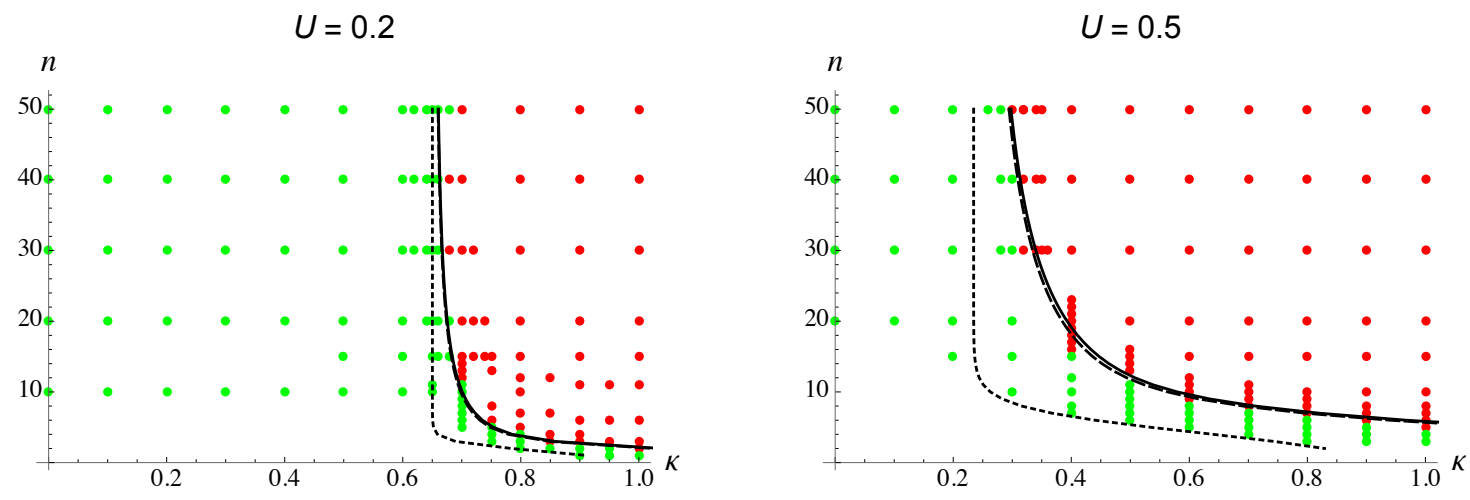

Figure 5. Evolution of self-fertilization under Gaussian stabilizing selection. The two

plots show the effect of the number of traits under selection $n$ and pollen discounting (parameter $\kappa$ ) on the evolution of self-fertilization for two values of the mutation rate on traits under stabilizing selection $(U=0.2$ and 0.5$)$. Green and red dots correspond to simulation results and have the same meaning as in the previous figures. The dotted curves show the maximum value of pollen discounting $\kappa$ for selfing to increase obtained when neglecting $\Delta_{\mathrm{LD}} \bar{\sigma}$ and $\Delta_{\text {purge }} \bar{\sigma}$ (that is, $\delta^{\prime}=(1-\kappa) / 2$ ), while the dashed and solid curves correspond to the predictions including the term $\Delta_{\mathrm{LD}} \bar{\sigma}$ (from equation 46) under free recombination (dashed) or integrated over the genetic map (solid). To obtain these predictions, the relation between $n$ and $\delta^{\prime}$ was obtained from a fit of the simulation results. Other parameter values: $\bar{\varsigma}=0.01, R=20$; in the simulations $N=5,000, U_{\text {self }}=0.001$ (overall mutation rate at selfing modifier loci), $\sigma_{\text {self }}=0.01$ (standard deviation of mutational effects on selfing). 